\title{
Initial serum creatinine concentration affects clinical outcomes in patients with IgA nephropathy treated with mycophenolate mofetil combined with low-dose prednisone
}

\author{
HAIYING SONG ${ }^{1,2^{*}}$, HAOFEI HU ${ }^{1,2^{*}}$, FEI TANG ${ }^{1,2^{*}}$, CHANGCHUN CAO $^{1,2}$, \\ QIJUN WAN ${ }^{1,2}$ and YONGCHENG HE Y,2 $^{1,2}$ \\ ${ }^{1}$ Department of Nephrology, The First Affiliated Hospital of Shenzhen University, Shenzhen Second People's Hospital; \\ ${ }^{2}$ Department of Nephrology, Shenzhen University Health Science Center, Shenzhen, Guangdong 518035, P.R. China
}

Received February 22, 2019; Accepted January 22, 2020

DOI: $10.3892 /$ etm.2020.8573

\begin{abstract}
Indicators for predicting the efficacy of mycophenolate mofetil (MMF) have so far remained elusive. The present study aimed to identify predictive indicators of the efficacy of MMF combined with low-dose prednisone in patients with IgA nephropathy. A total of 598 patients presenting with primary $\operatorname{Ig}$ A nephropathy at our center were screened. Patients were followed up for 18 months, where the end-point was defined as complete clinical remission. Cox proportional hazards models were performed for analyzing the initial serum creatinine ( $\mathrm{SCr}$ ) concentration to predict incomplete clinical remission. In total, 7 of 71 patients $(9.86 \%)$ were in complete clinical remission at the final visit. Logistic regression indicated that the hazard ratio (HR) for quartile 4 was significantly higher than the HR for quartile 1 (quartile 4 vs. quartile 1: HR, 2.51; 95\% CI, 1.20-5.21; $\mathrm{P}=0.01)$. Additional adjustment for the confounding variables, including age, sex, systolic BP, diastolic BP, proteinuria, uric acid, serum triglyceride, hemoglobin, serum albumin, serum total cholesterol and The Oxford classification of the models, did not reduce the HRs for the association between the initial SCr concentration and risk of incomplete clinical remission (quartile 4 vs. quartile 1: HR, 7.27; 95\% CI, 1.21-43.63; P=0.03). Each unit increase in the initial $\mathrm{SCr}$ concentration was associated with a 67 and 194\% increase in the risk of incomplete clinical remission based on model 1 (95\% CI, 1.02-2.73; $\mathrm{P}=0.04)$ and
\end{abstract}

Correspondence to: Professor Yongcheng He or Professor Qijun Wan, Department of Nephrology, The First Affiliated Hospital of Shenzhen University, Shenzhen Second People's Hospital, 3002 Sungang Road, Futian, Shenzhen, Guangdong 518035, P.R. China

E-mail: heyongcheng@medmail.com.cn

E-mail: yiyuan2224@sina.com

${ }^{*}$ Contributed equally

Key words: IgA nephropathy, mycophenolate mofetil, serum creatinine, clinical outcome model 2 (95\% CI, 1.01-8.60; P=0.048), respectively. In conclusion, in the present cohort of patients with $\operatorname{IgA}$ nephropathy treated with MMF plus low-dose prednisone, the initial $\mathrm{SCr}$ concentration was an independent risk factor for incomplete clinical remission.

\section{Introduction}

$\operatorname{Ig}$ A nephropathy $(\operatorname{Ig} \mathrm{AN})$ is the most common type of primary glomerulonephritis worldwide and is particularly prevalent in Asia (1). IgAN accounts for $45 \%$ of primary glomerulonephritis cases in China (2). IgAN causes end-stage renal disease (ESRD) in a significant proportion of patients (3-5). Given the devastating medical and socioeconomic burden for patients with chronic kidney disease, diverse therapeutic options should be considered to slow the progression of kidney disease $(6,7)$. As the pathogenic mechanisms of $\mathrm{IgAN}$ remain incompletely understood, no specific treatments remain unavailable. Most of the current treatment strategies include the regulation of blood pressure (BP) using angiotensin-converting enzyme inhibitors or angiotensin receptor blockers and amelioration of proteinuria.

Mycophenolate mofetil (MMF) is used as an immunosuppressant in patients undergoing renal transplantation. It is also used for immunosuppressive treatment in patients with autoimmune diseases $(8,9)$. MMF was first used to treat IgAN in 1997 (10). Certain prospective randomized controlled trials have proved the superior effectiveness of MMF to that of other immunosuppressive drugs or placebo in Chinese patients with less advanced $\operatorname{IgAN}(11,12)$. Although similar studies have been performed in $\mathrm{IgAN}$, little is known regarding which indicators are able to predict the efficacy of MMF combined with low-dose prednisone in $\operatorname{IgAN}$. Therefore, the aim of the present study was to identify predictive indicators of the efficacy of MMF combined with low-dose prednisone in IgAN.

\section{Patients and methods}

Study population. From September 2009 to October 2017, 598 patients with primary IgAN were screened at the Department of Nephrology of Shenzhen Second People's 
Hospital (Shenzhen, China). A total of 440 patients were excluded (comprising 400 patients who did not use MMF plus low-dose corticosteroids, 13 patients without baseline data and 27 patients were lost to follow-up). Subsequently, 158 patients using MMF plus low-dose corticosteroids were enrolled in the study. According to the literature (13), in vitro, the relative immunosuppressive potency of prednisone is zero, whereas that of methylprednisolone is 11 . Thus, patients treated with MMF plus methylprednisolone may be more susceptible to infection than those treated with MMF combined with prednisone. A retrospective study indicated that chronically impaired renal function and methylprednisolone treatment are risk factors for severe pneumonia. In patients with patients Lee Class III-V IgAN, MMF plus prednisone is safer than MMF plus methylprednisolone (14). In the present study, only indicators for predicting the efficacy of using MMF combined with low-dose prednisone in IgA nephropathy were investigated, and the 19 patients who used MMF plus low-dose methylprednisolone were therefore excluded. Furthermore, 26 patients who previously used immunosuppressive therapy were excluded, 18 patients who were lost to follow-up, 11 patients who withdrew from participation, 13 patients who were removed by the physician due to biopsy specimens containing $<8$ glomeruli. Finally, 71 patients with primary $\operatorname{IgAN}$ using MMF plus low-dose prednisone were included. The study selection process is depicted in Fig. 1. The key inclusion criteria were primary IgAN confirmed by biopsy and patient age of 18-70 years. The major exclusion criteria were as follows: i) Biopsy specimens containing $<8$ glomeruli; ii) secondary IgAN (e.g., caused by lupus, liver cirrhosis, Henoch-Schonlein purpura); and iii) any prior immunosuppressive therapy.

Study design and clinical, biochemical and histological data collection. A retrospective cohort study was performed. All patients enrolled underwent kidney biopsy from 2009 to 2017. Patients were followed up for 18 months unless they progressed to ESRD or severe pneumonia (within 18 months). Using a database from the Shenzhen Second People's Hospital, the demographic, clinical and biochemical data at the time of renal biopsy, including age, sex, systolic blood pressure (SBP) and diastolic blood pressure (DBP), were retrieved and considered baseline data. The following biochemical laboratory data were also collected: Proteinuria and concentrations of serum creatinine ( $\mathrm{SCr}$ ), hemoglobin, serum albumin, serum uric acid, serum triglycerides, serum total cholesterol, high-density lipoprotein cholesterol and low-density lipoprotein cholesterol. The estimated glomerular filtration rate was calculated using the Chronic Kidney Disease Epidemiology Collaboration equation (15).

The histological diagnosis of IgAN was based upon the demonstration of mesangial proliferative changes by light microscopy and the concomitant presence of predominant or codominant mesangial deposition of IgA. The pathological diagnosis was made by the same pathologist from Guangzhou King Medical Centre of Clinical Laboratory (Guangzhou, China). Renal lesions were histopathologically evaluated and scored using the Oxford classification (16). This classification identifies four pathological components: Mesangial hypercellularity (M), endocapillary hypercellularity (E), segmental glomerulosclerosis (S) and tubular atrophy/interstitial fibrosis (T). According to this classification, the following scoring system apply: i) Mesangial score $\leq 0.5$ (M0) or $>0.5$ (M1); ii) endocapillary hypercellularity absent (E0) or present (E1); iii) Segmental glomerulosclerosis absent (S0) or present (S1); iv) presence or absence of podocyte hypertrophy/tip lesions in biopsy specimens with S1; and v) Tubular atrophy/interstitial fibrosis $\leq 25 \%$ (T0), $26-50 \%$ (T1), or $>50 \%$ (T2) (16).

Treatments. The treatment regimen was MMF plus low-dose prednisone $(0.5 \mathrm{mg} / \mathrm{kg} / \mathrm{day})$. The initial dose of MMF was 1.0 or $1.5 \mathrm{~g} /$ day in patients with a body weight of $<50$ or $\geq 50 \mathrm{~kg}$, respectively. MMF therapy was initiated at $1-1.5 \mathrm{~g} /$ day for six months, 0.5-1.0 g/day for the next six months and then $0.25-0.5 \mathrm{~g} /$ day for the final six months. Prednisone was administered at $0.5 \mathrm{mg} / \mathrm{kg} / \mathrm{day}$ for two months and then slowly tapered by $5 \mathrm{mg}$ every two weeks until discontinuation. Patient records were reviewed from the start of treatment through to the time-point when the chart was reviewed and until the drug was discontinued or severe infection occurred (final time-point).

Follow-up and outcome measures. The data collected included age, sex, SBP, DBP, proteinuria, serum uric acid concentration, serum triglyceride concentration, hemoglobin concentration, serum albumin concentration, serum total cholesterol concentration and the Oxford classification. The information on the patients was retrieved from a database from Shenzhen Second People's Hospital and confirmed by a telephone call at the end of the study. The end-point was complete clinical remission (defined as proteinuria with a protein-to-creatinine ratio of $<0.2$ and stable renal function with a decrease in the estimated glomerular filtration rate (eGFR) of $<5 \mathrm{ml}$ per minute per $1.73 \mathrm{~m}^{2}$ from baseline at the end of the 18-month follow-up period) (17).

Statistical analysis. Continuous variables are expressed as the mean \pm standard deviation or median (range) as appropriate. The association of the initial SCr concentration was examined as a continuous variable categorized into quartiles with outcomes of incomplete clinical remission. Cox proportional hazards models were used to evaluate these associations without adjustment and with adjustment for confounding variables. In the adjusted regression model, age, sex, SBP, DBP, proteinuria, serum uric acid concentration, hemoglobin concentration, serum albumin concentration, serum triglyceride concentration, serum total cholesterol concentration and the Oxford classification were included. Hazard ratios (HRs) and $95 \%$ CIs were calculated. The lowest quartile was the reference for the initial $\mathrm{SCr}$ concentration.

Logistic regression analyses with the initial $\mathrm{SCr}$ concentration as a continuous variable were performed for the overall population. Interaction and stratified analyses were conducted according to sex (male and female), hypertension status at baseline (hypertension or no hypertension), endocapillary proliferation (E0 and E1) and segmental glomerulosclerosis/adhesion (S0 and S1). To examine the association between the initial $\mathrm{SCr}$ concentration and outcome, the Cox models were re-established using the initial $\mathrm{SCr}$ concentration as a continuous variable. HRs were calculated 


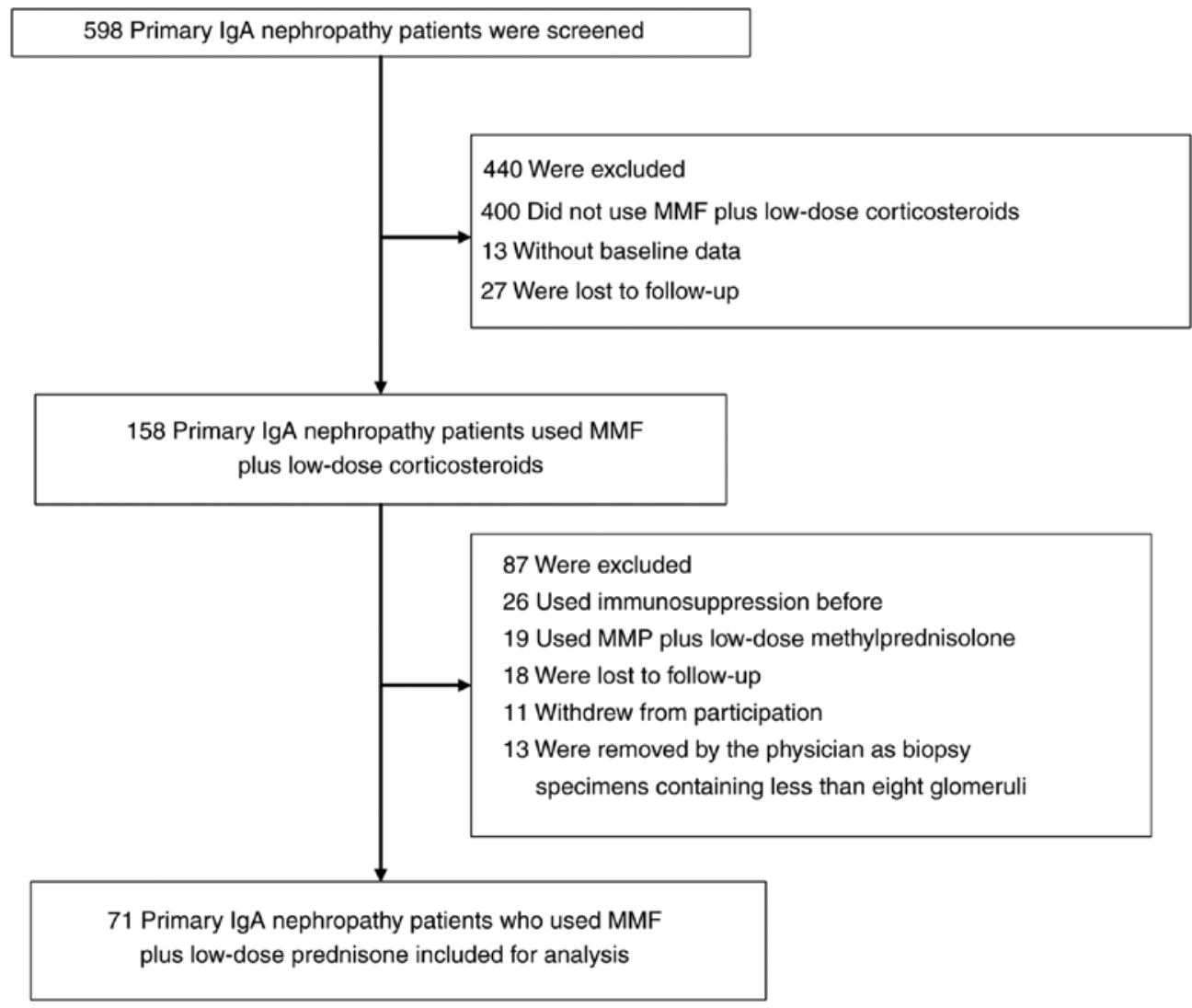

Figure 1. Eligibility and participation in the follow-up study. MMF, mycophenolate mofetil.

per $1 \mathrm{mg} / \mathrm{dl}$ increase in the initial $\mathrm{SCr}$ concentration and the risk change for incomplete clinical remission. The cutoff value for the initial $\mathrm{SCr}$ concentration was determined using receiver operating characteristics (ROC) curve analysis. Kaplan-Meier survival curves were used to compare complete clinical remission rates. All P-values were calculated using two-tailed tests of statistical significance with a type I error rate of $5 \%$. $\mathrm{P}$ interaction in the results section represents the $\mathrm{P}$-value for interaction. All statistical analyses were performed using Empower (R) (www.empowerstats.com; X\&Y Solutions, Inc.) and R (http://www.R-project.org).

\section{Results}

From September 2009 to October 2017, a total of 598 patients with primary IgAN presenting at the Department of Nephrology of Shenzhen Second People's Hospital (Shenzhen, China) were initially screened. As presented in the flow chart in Fig. 1, 440 patients were excluded (comprising 400 patients who did not use MMF plus low-dose corticosteroids, 13 patients without baseline data and 27 patients lost to follow-up). A total of 158 patients using MMF plus low-dose corticosteroids were retained. Of these, 87 patients were excluded (comprising 26 patients who previously used immunosuppressive therapy, 19 patients taking MMF plus low-dose methylprednisolone, 18 patients lost to follow-up, 11 patients who withdrew from participation and 13 patients removed by the physician as biopsy specimens contained $<8$ glomeruli). Finally, 71 patients with primary IgAN using MMF plus low-dose prednisone were included (Fig. 1).

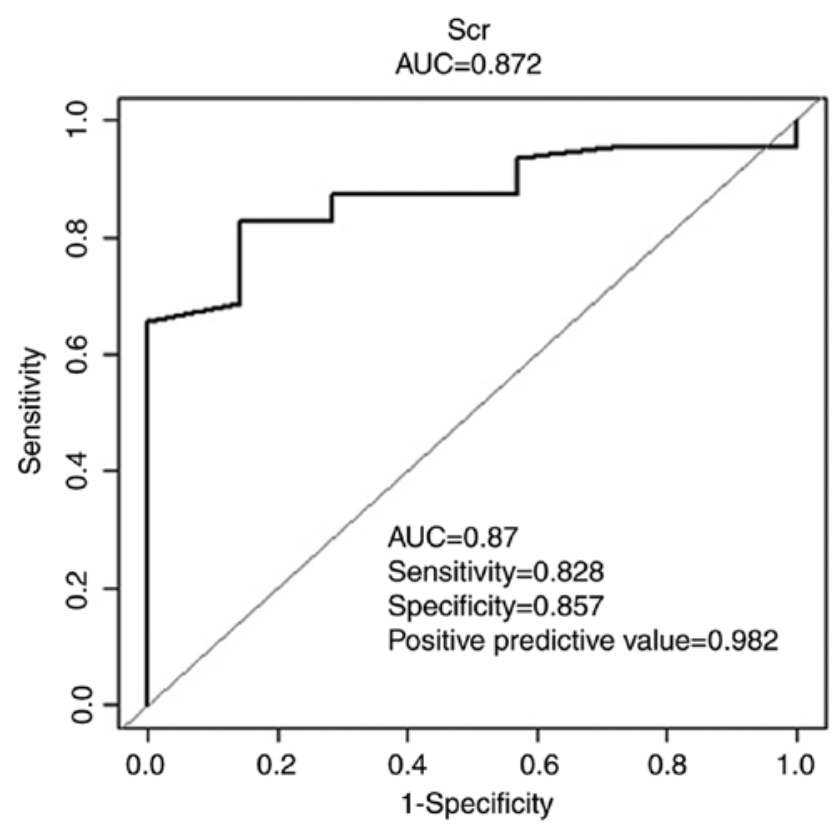

Figure 2. Receiver operating characteristic (ROC) curve for the initial $\mathrm{SCr}$ concentration. The cutoff value for the initial $\mathrm{SCr}$ concentration determined was calculated to be $0.89 \mathrm{mg} / \mathrm{dl}$. SCr, serum creatinine; AUC, area under curve.

The initial SCr concentration was the patient's SCr value at biopsy. The initial $\mathrm{SCr}$ concentration was the measurement prior to treatment with MMF combined with low-dose prednisone. The cutoff value for the initial SCr concentration determined using ROC curve analysis was 0.89 (Fig. 2). 
Table I. Patient characteristics at baseline.

\begin{tabular}{lccrr}
\hline Variable & $\begin{array}{c}\text { Low SCr group } \\
(<0.89 ; \mathrm{n}=16)\end{array}$ & $\begin{array}{c}\text { High SCr group } \\
(\geq 0.89 ; \mathrm{n}=55)\end{array}$ & Total $(\mathrm{n}=71)$ & P-value \\
\hline Age (years) & $34.25 \pm 9.57$ & $34.27 \pm 10.05$ & $34.27 \pm 9.87$ & 0.994 \\
Male & $1(6.25)$ & $31(56.36)$ & $32(45.07)$ & 0.063 \\
Hypertension & $12(75.00)$ & $29(52.73)$ & $41(57.75)$ & 0.112 \\
SBP $(\mathrm{mmHg})$ & $128.75 \pm 17.89$ & $139.91 \pm 20.84$ & $137.39 \pm 20.63$ & 0.038 \\
DBP $(\mathrm{mmHg})$ & $77.81 \pm 9.84$ & $88.60 \pm 14.17$ & $86.17 \pm 14.01$ & 0.006 \\
Serum creatinine $(\mathrm{mg} / \mathrm{dl})$ & $0.69 \pm 0.09$ & $1.60 \pm 0.65$ & $1.40 \pm 0.69$ & $<0.001$ \\
eGFR $\left(\mathrm{ml} / \mathrm{min}\right.$ per $\left.1.73 \mathrm{~m}^{2}\right)$ & $122.03 \pm 26.62$ & $56.16 \pm 22.05$ & $71.00 \pm 35.99$ & $<0.001$ \\
Proteinuria $(\mathrm{g} / 24 \mathrm{~h})$ & $1.04 \pm 0.74$ & $2.71 \pm 2.67$ & $2.26 \pm 2.42$ & 0.017 \\
Serum albumin $(\mathrm{g} / \mathrm{l})$ & $37.71 \pm 4.66$ & $37.71 \pm 6.17$ & $37.71 \pm 5.83$ & 0.996 \\
Uric acid $(\mu \mathrm{mol} / \mathrm{l})$ & $306.29 \pm 74.35$ & $447.06 \pm 102.19$ & $414.89 \pm 112.99$ & $<0.001$ \\
Hemoglobin $(\mathrm{g} / \mathrm{l})$ & $121.12 \pm 22.78$ & $126.57 \pm 22.41$ & $125.34 \pm 22.45$ & 0.397 \\
Triglycerides $(\mathrm{mmol} / \mathrm{l})$ & $1.17 \pm 0.68$ & $1.90 \pm 1.34$ & $1.72 \pm 1.25$ & 0.040 \\
Total cholesterol $(\mathrm{mmol} / \mathrm{l})$ & $4.59 \pm 0.88$ & $5.40 \pm 1.59$ & $5.20 \pm 1.49$ & 0.057 \\
HDLc $(\mathrm{mmol} / \mathrm{l})$ & $1.30 \pm 0.26$ & $1.19 \pm 0.32$ & $1.22 \pm 0.31$ & 0.224 \\
LDLc $(\mathrm{mmol} / \mathrm{l})$ & $2.66 \pm 0.69$ & $3.25 \pm 1.16$ & $3.11 \pm 1.09$ & 0.061 \\
The Oxford classification & & & & \\
M1 & $15(93.75)$ & $49(89.09)$ & $64(90.14)$ & 0.582 \\
E1 & $11(68.75)$ & $36(65.45)$ & $47(66.20)$ & 0.806 \\
S1 & $11(68.75)$ & $22(40.00)$ & $33(46.48)$ & 0.051 \\
T1 & $2(12.50)$ & $27(49.09)$ & $29(40.85)$ & $<0.001$ \\
T2 & $0(0.00)$ & $9(16.36)$ & $9(12.68)$ & $<0.001$ \\
\hline
\end{tabular}

Values are expressed as the mean \pm standard deviation, median (interquartile range) or $\mathrm{n}(\%)$. Hypertension was defined as blood pressure $\geq 140 / 90 \mathrm{mmHg}$ or on anti-hypertensive treatment. ${ }^{a}$ The Oxford classification: Mesangial score $\leq 0.5$ (M0) or $>0.5$ (M1). Endocapillary hypercellularity absent (E0) or present (E1). Segmental glomerulosclerosis absent (S0) or present (S1); presence or absence of podocyte hypertrophy/ tip lesions in biopsy specimens with S1. Tubular atrophy/interstitial fibrosis $\leq 25 \%$ (T0), 26-50\% (T1), or >50\% (T2). SBP, systolic blood pressure; DBP, diastolic blood pressure; SCr, serum creatinine; eGFR, estimated glomerular filtration rate; LDLc, low-density lipoprotein cholesterol; HDLc, high-density lipoprotein cholesterol.

Therefore, this level was selected to group patients. A total of 16 patients with an initial $\mathrm{SCr}$ concentration of $<0.89 \mathrm{mg} / \mathrm{dl}$ were assigned to the low $\mathrm{SCr}$ group, whereas 55 patients with an initial $\mathrm{SCr}$ concentration of $\geq 0.89 \mathrm{mg} / \mathrm{dl}$ were assigned to the high $\mathrm{SCr}$ group.

The 18-month trial was completed by 52 patients $(73.2 \%)$ in this study. In the full-analysis set, 7 of 71 patients $(9.86 \%)$ had achieved complete clinical remission at the final visit. Table I presents the baseline demographic, clinical and biochemical characteristics of the study subjects. The majority of the patients $(54.9 \%)$ were female. The mean age was $34.3 \pm 9.9$ years (range, $20-70$ years). The mean $\mathrm{SCr}$ concentration was $1.4 \pm 0.7 \mathrm{mg} / \mathrm{dl}$. The mean eGFR level was $71.0 \pm 36.0 \mathrm{ml} / \mathrm{min} / 1.73 \mathrm{~m}^{2}$. A total of 41 patients had an eGFR of at least $60 \mathrm{ml} / \mathrm{min} / 1.73 \mathrm{~m}^{2}$ and 21 patients had an eGFR between 30 and $59 \mathrm{ml} / \mathrm{min} / 1.73 \mathrm{~m}^{2}$. In addition, patients in the low $\mathrm{SCr}$ group had less proteinuria, higher eGFR, lower triglyceride concentrations and lower uric acid concentrations compared with patients in the high $\mathrm{SCr}$ group (Table I). Kaplan-Meier survival analysis revealed that patients in the high $\mathrm{SCr}$ group had a significantly lower rate of complete clinical remission than those in the low $\mathrm{SCr}$ group (Fig. 3). Table II presents the results of the logistic regression analysis using univariate and multivariate Cox proportional hazards models. In the univariate analysis, the HR for incomplete clinical remission significantly increased as the quartiles of the initial $\mathrm{SCr}$ concentration increased. The HR for quartile 4 was significantly higher than the HR for quartile 1 (quartile 4 vs. quartile 1: HR, 2.51; 95\% CI, 1.20-5.21; $\mathrm{P}=0.01$ ). Additional adjustment for confounding variables did not reduce the HRs for the association between the initial SCr concentration and incomplete clinical remission (quartile 4 vs. quartile 1: HR, 7.27; 95\% CI, 1.21-43.63; $\mathrm{P}=0.03$ ). The total data were analyzed, where each unit increase in the initial $\mathrm{SCr}$ concentration was associated with a 67 and 194\% increase in the risk of incomplete clinical remission based on model 1 (95\% CI, 1.02-2.73; $\mathrm{P}=0.04)$ and model 2 (95\% CI, 1.01-8.60; P=0.048), respectively. Following adjustment for age, sex, proteinuria, Oxford classification, SBP and DBP during follow-up, the HRs for the association between the initial $\mathrm{SCr}$ concentration and incomplete clinical remission also increased.

To determine the consistency in the association between increased initial SCr concentration and the risk of incomplete clinical remission, interaction and stratified analyses were performed (Table III). For each unit increase in the initial $\mathrm{SCr}$ concentration, the HR for incomplete clinical remission was 
Table II. Logistic regression analysis of the association between the initial serum creatinine concentration and the risk of incomplete clinical remission.

\begin{tabular}{lcccccc}
\hline Group & $\begin{array}{c}\text { Unadjusted Hazard } \\
\text { ratio }(95 \% \mathrm{CI})\end{array}$ & P-value & $\begin{array}{c}\text { Model } 1^{\mathrm{a}} \text { Hazard } \\
\text { ratio }(95 \% \mathrm{CI})\end{array}$ & P-value & $\begin{array}{c}\text { Model } \mathrm{b}^{\mathrm{b}} \text { Hazard } \\
\text { ratio }(95 \% \mathrm{CI})\end{array}$ & P-value \\
\hline Total & $1.40(1.01-1.94)$ & 0.041 & $1.67(1.02=2.73)$ & 0.040 & $2.94(1.01=8.60)$ & 0.048 \\
Quartiles & & & & & & \\
$\quad$ Quartile 1 $(<0.90)$ & 1.0 & & 1.0 & & 1.0 & \\
Quartile 2 $(\geq 0.90-<1.25)$ & $1.70(0.80-3.58)$ & 0.167 & $2.16(0.73=6.39)$ & 0.166 & $1.69(0.45=6.31)$ & 0.435 \\
Quartile 3 $(\geq 1.25-<1.64)$ & $1.84(0.89=3.83)$ & 0.101 & $2.22(0.78=6.27)$ & 0.133 & $2.29(0.63=8.31)$ & 0.209 \\
Quartile 4 $(\geq 1.64)$ & $2.51(1.204=5.210)$ & 0.014 & $6.18(1.71=22.36)$ & 0.006 & $7.27(1.21=43.63)$ & 0.030 \\
\hline
\end{tabular}

Models: aModel adjusted for age, sex, systolic BP, diastolic BP, proteinuria, uric acid, serum triglyceride; ${ }^{\mathrm{b}}$ model adjusted as for model 1 plus hemoglobin, serum albumin, serum total cholesterol and The Oxford classification. BP, blood pressure.

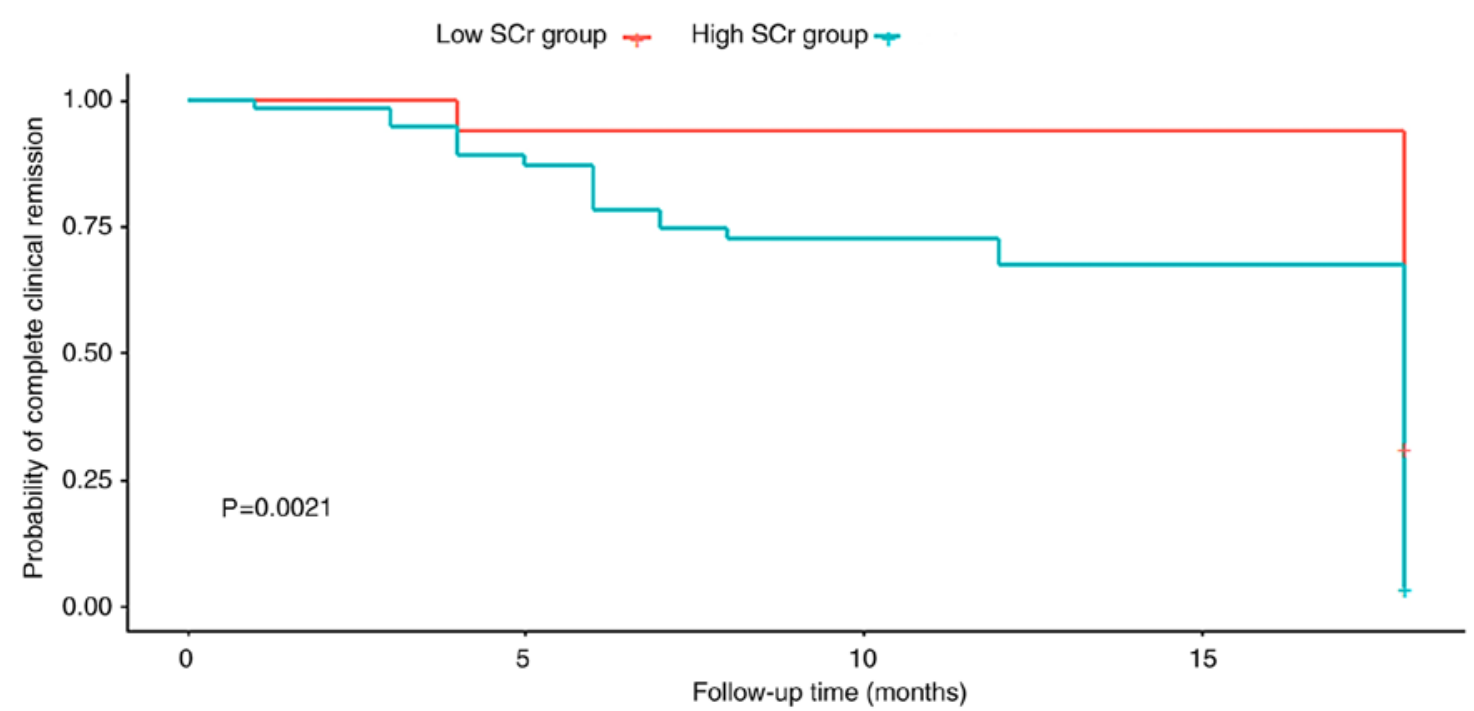

Figure 3. Kaplan-Meier curves for the rate of complete clinical remission according to the initial SCr concentration in patients with primary IgAN treated with mycophenolate mofetil plus low-dose prednisone. SCr, serum creatinine.

1.16 in males $(\mathrm{P}=0.60)$ and 1.55 in females $(\mathrm{P}=0.04)$, and the difference was not statistically significant $(\mathrm{P}$ interaction $=0.41)$. The HR for incomplete clinical remission for patients in the E1 subgroup was $2.09(\mathrm{P}=0.02)$ compared with $1.33(\mathrm{P}=0.15)$ for patients in the E0 subgroup ( $\mathrm{P}$ interaction $=0.24)$. The HR for patients in the $\mathrm{S} 1$ subgroup was $1.95(\mathrm{P}=0.007)$ and that for patients in the $\mathrm{S} 0$ subgroup was $1.10(\mathrm{P}=0.70)$. There were no statistically significant differences in patients stratified by $M$ or $\mathrm{T}$ (data not shown). In addition, for each unit increase in the initial SCr concentration, the HR for incomplete clinical remission was $1.44(\mathrm{P}=0.06)$ and $1.35(\mathrm{P}=0.19)$ for hypertensive and normotensive patients, respectively.

\section{Discussion}

IgAN is the leading cause of ESRD (18). There are substantial ethnic variations and Asian populations have an increased incidence of $\operatorname{IgAN}$ and an elevated risk of renal function decline (19). The predominant characteristic of $\operatorname{IgAN}$ is galactose-deficient $\operatorname{IgA} 1$ deposition in the mesangial area. However, the pathogenesis of $\operatorname{Ig} \mathrm{AN}$ is still not fully understood and clinical and pathological phenotypes of IgAN are variable. Thus, treatments for IgAN have not been effective. MMF acts by releasing mycophenolic acid, which leads to apoptosis in cytotoxic T-lymphocytes and reduction in antibody synthesis via the selective inhibition of T- and B-lymphocyte proliferation $(20,21)$, induction of intercellular adhesion molecule-1 mRNA expression and interleukin-6 secretion, and inhibition of cell proliferation, particularly in endothelial cells $(22,23)$. MMF is beneficial for IgAN secondary to systemic diseases, including lupus nephritis and hepatitis B virus-associated glomerulonephritis $(24,25)$. Certain studies have indicated that in diffuse proliferative lupus nephritis, MMF has a higher efficacy to induce remission than pulsed intravenous therapy with cyclophosphamide (CTX) (26-28). To date, eight randomized controlled trials on MMF therapy for IgAN have been performed. Certain studies demonstrated no benefit from MMF therapy (29-31), whereas other studies observed amelioration of proteinuria and long-term renoprotection following treatment with MMF $(11,32)$.

An Italian multicenter study indicated that in patients with active disease, proteinuria $>2.4 \mathrm{~g}$ /day and renal failure 
Table III. Hazard ratios with $95 \%$ CIs for incomplete clinical remission per unit increase in the initial serum creatinine concentration in subgroups by sex, hypertension, E and S.

\begin{tabular}{lcccc}
\hline Item & $\begin{array}{c}\text { Cases } \\
(\mathrm{n})\end{array}$ & $\begin{array}{c}\text { Hazard ratio } \\
(95 \% \mathrm{CI})\end{array}$ & P-value & $\begin{array}{c}\text { P for } \\
\text { interaction }\end{array}$ \\
\hline Sex & & & & 0.414 \\
$\quad$ Male & 32 & $1.16(0.67-2.02)$ & 0.601 & \\
$\quad$ Female & 39 & $1.55(1.02-2.36)$ & 0.042 & \\
Hypertension & & & & 0.850 \\
$\quad$ Yes & 41 & $1.44(0.85-2.45)$ & 0.061 & \\
No & 30 & $1.35(0.86-2.10)$ & 0.189 & \\
E & & & & 0.242 \\
E0 & 24 & $1.33(0.90-1.97)$ & 0.151 & \\
E1 & 47 & $2.09(1.12-3.91)$ & 0.020 & \\
S & & & & 0.096 \\
S0 & 38 & $1.10(0.69-1.75)$ & 0.697 & \\
S1 & 33 & $1.95(1.20-3.17)$ & 0.007 & \\
\hline
\end{tabular}

Hypertension was defined as blood pressure $\geq 140 / 90 \mathrm{mmHg}$ or on anti-hypertensive treatment. E, endocapillary proliferation; $\mathrm{S}$, segmental glomerulosclerosis/adhesion. E0, endocapillary hypercellularity absent; E1, endocapillary hypercellularity present. S0, segmental glomerulosclerosis absent; S1, segmental glomerulosclerosis present; presence or absence of podocyte hypertrophy/tip lesions in biopsy specimens with $\mathrm{S} 1$.

(mean SCr concentration, $1.6 \mathrm{mg} / \mathrm{dl}$ ), a combined regimen with MMF and steroids may induce amelioration of proteinuria and delay successive progression toward renal failure (33). A Chinese study compared combined therapy with prednisone and MMF vs. prednisone and CTX for severe $\operatorname{IgAN}$. In the MMF group, amelioration of proteinuria and improvement of renal function were observed, with fewer adverse events than in the CTX group (34). However, another study evaluated the efficacy and safety of MMF plus prednisone vs. full-dose prednisone in patients with IgAN with active proliferative lesions. At 6 months, there were no significant differences between the groups regarding complete remission or median time to achieve remission. In addition, during the follow-up period, relapse rates and total adverse events did not differ between the groups (35). These mentioned studies enrolled patients with different clinical and histological characteristics. For the various patients with different clinical and histological characteristics, the efficacy of MMF therapy exhibited certain differences. It is necessary to identify indicators for predicting the efficacy of using MMF in patients with IgAN. Therefore, in the present study, predictive indicators of the efficacy of using mycophenolate mofetil combined with low-dose prednisone in IgAN patients were determined.

In the present retrospective cohort study, $\sim 10 \%$ of patients (7/71) displayed complete clinical remission with MMF plus prednisone. The rate of complete clinical remission for patients using MMF plus low-dose prednisone was further determined and it was revealed that it was possible to predict the efficacy of using MMF combined with low-dose prednisone using the initial SCr concentration. During the course of treatment with MMF plus low-dose prednisone, the average SCr value of the patients with complete clinical remission was relatively stable and its changes occurred slowly. However, the variations in the average $\mathrm{SCr}$ value of the patients with incomplete clinical remission were significant. The variations in the average $\mathrm{SCr}$ value of the patients with incomplete clinical remission were $\sim 5$ times higher than those of the patients with complete clinical remission. A higher initial SCr concentration was significantly associated with an increased incidence of incomplete clinical remission. This association persisted even after adjustment for demographic, clinical and histopathology factors.

The following clinical predictors of renal outcome in IgAN, determined at the time of diagnosis, have been assessed in several clinical studies: Proteinuria, hypertension, decreased eGFR (36-38) and histological grading (39). Certain risk-prediction tools indicated that in $\operatorname{IgA}$ nephropathy, the risk factors included age, sex, the Oxford classification histologic score, serum albumin and serum uric acid $(40,41)$. Furthermore, studies have indicated that serum triglyceride, serum total cholesterol and hemoglobin were risk factors for the prognosis of IgA nephropathy $(42,43)$. Therefore, these variables were adjusted to minimize the potential impact of confounders. Of note, in the present study, the Oxford classification was not independently associated with the occurrence of incomplete clinical remission. The present results will thus be important for determining therapeutic strategies in the clinic.

There has been a great interest in exploring the association between clinical markers and renal outcomes. In a study of 113 Chinese patients diagnosed with crescentic IgAN, the initial SCr concentration was the strongest predictor of kidney failure (44). Significant associations between SCr concentration and renal prognosis in patients with IgAN have also been demonstrated. However, there are limited data on the association between the initial SCr concentration and the efficacy of MMF combined with low-dose prednisone in IgAN. The present results are consistent with those of a previous study (45), indicating that patients with a higher initial $\mathrm{SCr}$ concentration had a higher risk of incomplete clinical remission after adjustment for other risk factors. In the present study, stratified analyses suggested that the risk of incomplete clinical remission increased significantly with increasing initial SCr concentrations for female patients but not for male patients. These results were consistent with those of previous studies. A number of large-scale studies have indicated that the rate of progression of renal disease is greater in females than in males $(46,47)$. Furthermore, the risk of incomplete clinical remission increased significantly with increasing initial $\mathrm{SCr}$ concentrations for patients in the E1 and S1 subgroups. This result was consistent with the Oxford classification, which may aid in predicting outcomes for patients with $\operatorname{IgAN}(16,48)$.

Of note, the present study had certain important limitations that should be noted. First, the number of observed events is small and limits the statistical power of this explorative study to a certain extent. However, according to a recent meta-analysis, eight randomized controlled trials have assessed the use of MMF in patients with IgA nephropathy $(11,12,28-31,33,49,50)$. Most of them were also small-sized and the study with the biggest size included 84 patients (33). The patients in these studies and the present study are similar in number. Furthermore, 
statistical methods were applied to retrospectively determine predictive indicators of the efficacy of using MMF combined with low-dose prednisone in patients with $\operatorname{Ig} \mathrm{AN}$. Of note, the present results were statistically significant and the sample size was sufficient to draw a conclusion. Furthermore, the Oxford or MEST classification was used, while crescentic (C) features were not considered. The IgA Nephropathy Classification Working Group provided an update that $\mathrm{C}$ features should be added to the MEST score and biopsy reporting should provide a MEST-C score for the previous year (51). In addition, the present study is based on a cohort from a single center, and whether these observations may be extrapolated to other populations of Chinese and non-Chinese IgAN patients using MMF plus prednisone remains to be evaluated.

In conclusion, in the present cohort of patients with $\mathrm{IgAN}$ treated with MMF plus low-dose prednisone, the initial $\mathrm{SCr}$ concentration was an independent risk factor for the outcome of incomplete clinical remission. The present study is important for determining therapeutic strategies in the clinic. Further research is required to replicate these results in other cohorts and to compare their predictive ability with that of traditional factors, including proteinuria, hypertension and the new Oxford classification.

\section{Acknowledgements}

Not applicable.

\section{Funding}

This study was supported in part by the Basic Research Project and Technology Development Projects of Shenzhen Municipal Science and Technology Innovation Council (grant nos. JCYJ20130329104904512, CXZZ20140421155346007 and CXZZ20150601140615135) and the Research Project of the Health and Family Planning Commission of Shenzhen Municipality (grant no. SZFZ2018063).

\section{Availability of data and materials}

The datasets used and/or analyzed during the present study available from the corresponding author on reasonable request.

\section{Authors' contributions}

YH, QW, HS, HH and FT contributed to the study conception and design, analyzed and interpreted the data and drafted the manuscript. CC analyzed data and reviewed the manuscript. $\mathrm{HS}$ and $\mathrm{HH}$ oversaw the project, contributed to the discussion and reviewed the manuscript. $\mathrm{YH}$ and $\mathrm{QW}$ are the guarantors of this work and, as such, had full access to all the data in the study and take responsibility for the integrity of the data and the accuracy of the data analysis. All authors read and approved the final version of the manuscript.

\section{Ethics approval and consent to participate}

This study was approved by the Review Board and Ethics Committee of Shenzhen Second People's Hospital (no. 20180110010; Shenzhen, China). Although all patients included in this study were provided with the description of investigations, the study was performed as a medical record-based retrospective analysis and the included subjects were anonymized. Therefore, the Review Board and Ethics Committee of Shenzhen Second People's Hospital approved the exemption from obtaining written consent (no. 20180110010).

\section{Patient consent for publication}

Not applicable.

\section{Competing interests}

The authors declare that they have no competing interests.

\section{References}

1. D'Amico G: Natural history of idiopathic IgA nephropathy and factors predictive of disease outcome. Semin Nephrol 24: 179-196, 2004.

2. Li LS and Liu ZH: Epidemiologic data of renal diseases from a single unit in China: Analysis based on 13,519 renal biopsies. Kidney Int 66: 920-923, 2004.

3. Koyama A, Igarashi $M$ and Kobayashi M: Natural history and risk factors for immunoglobulin A nephropathy in Japan. Research Group on Progressive Renal Diseases. Am J Kidney Dis 29: 526-532, 1997.

4. D'Amico G: Natural history of idiopathic IgA nephropathy: Role of clinical and histological prognostic factors. Am J Kidney Dis 36: 227-237, 2000

5. Donadio JV and Grande JP: IgA nephropathy. N Engl J Med 347: 738-748, 2002.

6. Collins AJ, Foley RN, Gilbertson DT and Chen SC: United States Renal Data System public health surveillance of chronic kidney disease and end-stage renal disease. Kidney Int Suppl (2011) 5: 2-7, 2015.

7. Jin DC, Yun SR, Lee SW, Han SW, Kim W, Park J and Kim YK: Lessons from 30 years' data of Korean end-stage renal disease registry, 1985-2015. Kidney Res Clin Pract 34: 132-139, 2015.

8. Ginzler EM, Dooley MA, Aranow C, Kim MY, Buyon J, Merrill JT, Petri M, Gilkeson GS, Wallace DJ, Weisman MH and Appel GB: Mycophenolate mofetil or intravenous cyclophosphamide for lupus nephritis. N Engl J Med 353: 2219-2228, 2005

9. Rasche FM, Keller F, von Muller L, Sailer LK, Karges W and Czock D: Mycophenolic acid therapy after cyclophosphamide pulses in progressive IgA nephropathy. J Nephrol 19: 465-472, 2006.

10. Nowack R, Birck R and van der Woude FJ: Mycophenolate mofetil for systemic vasculitis and IgA nephropathy. Lancet 349: 774, 1997.

11. Tang S, Leung JC, Chan LY, Lui YH, Tang CS, Kan CH, Ho YW and Lai KN: Mycophenolate mofetil alleviates persistent proteinuria in IgA nephropathy. Kidney Int 68: 802-812, 2005.

12. Chen X, Chen P, Cai G, Wu J, Cui Y, Zhang Y, Liu S and Tang L: A randomized control trial of mycophenolate mofetil treatment in severe IgA nephropathy. Zhonghua Yi Xue Za Zhi 82: 796-801, 2002 (In Chinese).

13. Langhoff E, Olgaard K and Ladefoged J: The immunosuppressive potency in vitro of physiological and synthetic steroids on lymphocyte cultures. Int J Immunopharmacol 9: 469-473, 1987.

14. Wan QJ, Hu HF, He YC, Luan SD, Chen HT, Liu HP, Li T, Xu Y, $\mathrm{Xu} \mathrm{HL}$ and Liao Y: Severe pneumonia in mycophenolate mofetil combined with low-dose corticosteroids-treated patients with immunoglobulin A nephropathy. Kaohsiung J Med Sci 31: 42-46, 2015.

15. Levey AS, Stevens LA, Schmid CH, Zhang YL, Castro AF III, Feldman HI, Kusek JW, Eggers P, Van Lente F, Greene T, et al: A new equation to estimate glomerular filtration rate. Ann Intern Med 150: 604-612, 2009.

16. Working Group of the International IgA Nephropathy Network and the Renal Pathology Society; Cattran DC, Coppo R, Cook HT, Feehally J, Roberts IS, Troyanov S, Alpers CE, Amore A, Barratt J, et al: The Oxford classification of IgA nephropathy: Rationale, clinicopathological correlations, and classification. Kidney Int 76: 534-545, 2009. 
17. Rauen T, Eitner F, Fitzner C, Sommerer C, Zeier M, Otte B, Panzer U, Peters H, Benck U, Mertens PR, et al: Intensive supportive care plus immunosuppression in IgA nephropathy. N Engl J Med 373: 2225-2236, 2015.

18. Moresco RN, Speeckaert MM and Delanghe JR: Diagnosis and monitoring of IgA nephropathy: The role of biomarkers as an alternative to renal biopsy. Autoimmun Rev 14: 847-853, 2015.

19. Barbour SJ, Cattran DC, Kim SJ, Levin A, Wald R, Hladunewich MA and Reich HN: Individuals of pacific Asian origin with IgA nephropathy have an increased risk of progression to end-stage renal disease. Kidney Int 84: 1017-1024, 2013.

20. Allison AC and Eugui EM: Mycophenolate mofetil and its mechanisms of action. Immunopharmacology 47: 85-118, 2000.

21. Allison AC and Eugui EM: Purine metabolism and immunosuppressive effects of mycophenolate mofetil (MMF). Clin Transpl 10: 77-84, 1996.

22. Rosselli JL, Thacker SM, Karpinski JP and Petkewicz KA: Treatment of IgA nephropathy: An update. Nephrology 45: 1284-1296, 2011

23. Huang Y, Liu Z, Huang H, Liu H and Li L: Effects of mycophenolic acid on endothelial cells. Int Immunopharmacol 5 : 1029-1039, 2005

24. Rathi M, Goyal A, Jaryal A, Sharma A, Gupta PK, Ramachandran R, Kumar V, Kohli HS, Sakhuja V, Jha V, et al: Comparison of low-dose intravenous cyclophosphamide with oral mycophenolate mofetil in the treatment of lupus nephritis. Kidney Int 89: 235-242, 2016.

25. Zheng XY, Wei RB, Tang L, Li P and Zheng XD: Meta-analysis of combined therapy for adult hepatitis B virus-associated glomerulonephritis. World J Gastroenterol 18: 821-832, 2012

26. Zhu B, Chen N, Lin Y, Ren H, Zhang W, Wang W, Pan X and $\mathrm{Yu} \mathrm{H}$ : Mycophenolate mofetil in induction and maintenance therapy of severe lupus nephritis: A meta-analysis of randomized controlled trials. Nephrol Dial Transplant 22: 1933-1942, 2007.

27. Ong LM, Hooi LS, Lim TO, Goh BL, Ahmad G, Ghazalli R, Teo SM, Wong HS, Tan SY, Shaariah W, et al: Randomized controlled trial of pulse intravenous cyclophosphamide versus mycophenolate mofetil in the induction therapy of proliferative lupus nephritis. Nephrology 10: 504-510, 2005.

28. Laskari K, Mavragani CP, Tzioufas AG and Moutsopoulos HM: Mycophenolate mofetil as maintenance therapy for proliferative lupus nephritis: A longterm observational prospective study. Arthritis Res Ther 12: R208, 2010.

29. Maes BD, Oyen R, Claes K, Evenepoel P, Kuypers D Vanwalleghem J, Van Damme B and Vanrenterghem YF: Mycophenolate mofetil in IgA nephropathy: Results of a 3-year prospective placebo-controlled randomized study. Kidney Int 65 : $1842-1919,2004$

30. Frisch G, Lin J, Rosenstock J, Markowitz G, D'Agati V Radhakrishnan J, Preddie D, Crew J, Valeri A and Appel G: Mycophenolate mofetil (MMF) vs placebo in patients with moderately advanced IgA nephropathy: A doubleblind randomized controlled trial. Nephrol Dial Transplant 20: 2139-2145, 2005.

31. Hogg RJ, Bay RC, Jennette JC, Sibley R, Kumar S, Fervenza FC, Appel G, Cattran D, Fischer D, Hurley RM, et al: Randomised controlled trial of mycophenolate mofetil in children, adolescents, and adults with IgA nephropathy. Am J Kidney Dis 66 : 783-791, 2015

32. Tang SC, Tang AW, Wong SS, Leung JC, Ho YW and Lai KN: Long-term study of mycophenolate mofetil treatment in $\operatorname{IgA}$ nephropathy. Kidney Int 77: 543-549, 2010.

33. Roccatello D, Rossi D, Marletto F, Naretto C, Sciascia S, Baldovino S, Piras D and Giachino O: Long term effects of methylprednisolone pulses and mycophenolate mofetil in Ig A nephropathy patients at risk of progression. J Nephrol 25 $198-203,2012$
34. Liu X, Dewei D, Sun S, Xu G, Liu H, He L and Zhang P: Treatment of severe IgA nephropathy: Mycophenolate mofetil/prednisone compared to cyclophosphamide/prednisone. Int J Clin Pharmacol Ther 52: 95-102, 2014.

35. Hou JH, Le WB, Chen N, Wang WM, Liu ZS, Liu D, Chen JH, Tian J, Fu P, Hu ZX, et al: Mycophenolate mofetil combined with prednisone versus full-dose prednisone in IgA nephropathy with active proliferative lesions: A randomized controlled trial. Am J Kidney Dis 69: 788-795, 2017.

36. Wyatt RJ and Julian BA: IgA nephropathy. N Engl J Med 368: 2402-2414, 2013

37. Maixnerova D, Bauerova L, Skibova J, Rysava R, Reiterova J, Merta M, Honsova E and Tesar V: The retrospective analysis of 343 Czech patients with IgA nephropathy-one centre experience. Nephrol Dial Transplant 27: 1492-1498, 2012.

38. Reich HN, Troyanov S, Scholey JW and Cattran DC; Toronto Glomerulonephritis Registry: Remission of proteinuria improves prognosis in IgA nephropathy. J Am Soc Nephrol 18: 3177-3183, 2007.

39. Alamartine E, Sauron C, Laurent B, Sury A, Seffert A and Mariat C: The use of Oxford classification of $\operatorname{IgA}$ nephropathy to predict renal survival. Clin J Am Soc Nephrol 6: 2384-2388, 2011.

40. Barbour SJ, Coppo R, Zhang H, Liu ZH, Suzuki Y, Matsuzaki K, Katafuchi R,Er L, Espino-Hernandez G, Kim SJ, et al: Evaluating a new international risk-prediction tool in IgA nephropathy. JAMA Intern Med 179: 942-952, 2019.

41. Chen T, Li X, Li Y, Xia E, Qin Y, Liang S, Xu F, Liang D, Zeng C and Liu Z: Prediction and risk stratification of kidney outcomes in IgA nephropathy. Am J Kidney Dis 74: 300-309, 2019.

42. Syrjänen J, Mustonen J and Pasternack A: Hypertriglyceridaemia and hyperuricaemia are risk factors for progression of $\operatorname{Ig} \mathrm{A}$ nephropathy. Nephrol Dial Transplant 15: 34-42, 2000.

43. Shu D, Xu F, Su Z, Zhang J, Chen C, Zhang J, Ding X, Lv Y, Lin $\mathrm{H}$ and Huang P: Risk factors of progressive IgA nephropathy which progress to end stage renal disease within ten years: A case-control study. BMC Nephrol 18: 11, 2017.

44. Lv J, Yang Y, Zhang H, Chen W, Pan X, Guo Z, Wang C, Li S, Zhang J, Zhang J, et al: Prediction of outcomes in crescentic $\operatorname{Ig} \mathrm{A}$ nephropathy in a multicenter cohort study. J Am Soc Nephrol 24: 2118-2125, 2013

45. Ni Z, Yuan Y, Wang Q, Cao L, Che XJ, Zhang MF, Xie YY, Qi CJ and Mou S: Time-averaged albumin predicts the long-term prognosis of IgA nephropathy patients who achieved remission. J Transl Med 12: 194, 2014.

46. Haroun MK, Jaar BG, Hoffman SC, Comstock GW, Klag MJ and Coresh J: Risk factors for chronic kidney disease: A prospective study of 23,534 men and women in Washington County, Maryland. J Am Soc Nephrol 14: 2934-2941, 2003.

47. Zoppini G, Targher G, Chonchol M, Perrone F, Lippi G and Muggeo M: Higher HDL cholesterol levels are associated with a lower incidence of chronic kidney disease in patients with type 2 diabetes. Nutr Metab Cardiovasc Dis 19: 580-586, 2009.

48. Herzenberg AM, Fogo AB, Reich HN, Troyanov S, Bavbek N, Massat AE, Hunley TE, Hladunewich MA, Julian BA, Fervenza FC, et al: Validation of the Oxford classification of IgA nephropathy. Kidney Int 80: 310-317, 2011.

49. Liu XW, Li DM, Xu GS and Sun SR: Comparison of the therapeutic effects of leflunomide and mycophenolate mofetil in the treatment of immunoglobulin a nephropathy manifesting with nephrotic syndrome. Int J Clin Pharmacol Ther 48: 509-513, 2010

50. Du B, Jia Y, Zhou W, Min X, Miao L and Cui W: Efficacy and safety of mycophenolate mofetil in patients with IgA nephropathy: An update meta-analysis. BMC Nephrol 18: 245, 2017.

51. Trimarchi H, Barratt J, Cattran DC, Cook HT, Coppo R, Haas M, Liu ZH, Roberts IS, Yuzawa Y, Zhang H, et al: Oxford Classification of Ig A nephropathy 2016-the role of crescentic lesions: An update from the IgA Nephropathy Classification Working Group. Kidney Int 91: 1014-1021, 2017. 